\title{
Controllable spin pairing states in silicene-based superconducting hybrid structures with noncollinear magnetizations
}

\author{
Yajun Wei, ${ }^{1}$ Tinmin Liu, ${ }^{1}$ Chuanshuai Huang, ${ }^{1}$ Y. C. Tao $\odot,{ }^{1, *}$ and Fenghua $\mathrm{Qi}^{2, \dagger}$ \\ ${ }^{1}$ Department of Physics and Institute of Theoretical Physics, Nanjing Normal University, Nanjing 210023, China \\ ${ }^{2}$ School of Electronic Engineering, Nanjing Xiaozhuang University, Nanjing 211171, China
}

(Received 27 May 2021; revised 18 July 2021; accepted 20 July 2021; published 9 August 2021)

\begin{abstract}
We have theoretically investigated the modulation between the opposite- and equal-spin pairings and the related transport properties in silicene-based ferromagnet/ferromagnet/superconductor hybrid structures with noncollinear magnetizations. Due to the exotic electronic properties of silicene, the exclusive fully spin-polarized equal-spin pairing state appears without any contamination from the opposite-spin pairing state in a perpendicular magnetic configuration. Furthermore, the switch effect between fully spin-polarized opposite- and equal-spin pairings can be realized by tuning the Fermi level. In addition, the fully spin-polarized equal-spin pairing correlation can be enhanced by modulation of the magnitude and orientation of the exchange field in the central region. It is also a significant finding that for the formation of the fully spin-polarized equal-spin pairing state, the length of the central layer can be taken to be large, but not for opposite-spin pairing. Our findings provide an ideal platform to explore fully spin-polarized opposite- and equal-spin pairing states separately.
\end{abstract}

DOI: 10.1103/PhysRevResearch.3.033131

\section{INTRODUCTION}

With the advent of graphene and topological insulators [1,2], the study of Dirac fermions has become one of the most active research fields in condensed matter systems due to their exotic physical properties and potential applications over the past decade. Recently, as the analog of graphene, silicene has been attracting much attention both theoretically and experimentally [3-8]. However, there are two significant differences between graphene and silicene. One is that, unlike the flat sheet of graphene, silicene has a low buckling structure with a honeycomb lattice, where its two sublattices are separated by a perpendicular distance [8,9]. The other is that silicene has a much stronger spin-orbit coupling $[5,10,11]$, which results in a larger energy gap $[12,13]$. Moreover, this prototypical two-dimensional material has been grown experimentally by depositing silicene sheets on various substrates such as $\mathrm{Ag}(111), \mathrm{ZrB}_{2}(0001), \operatorname{Ir}(111)$, and $\mathrm{MoS}_{2}$ [6,8,14-17]. Silicene has multifarious future applications ranging from spintronics [18-22] to silicene-based transistors [23] at room temperature. There has been some theoretical research on proximity-induced ferromagnetism and superconductivity in silicene as well, which opens a realm of study on the transport properties in silicene-based single- and multiinterface superconducting hybrid structures [24-26].

There is phase-coherent scattering known as Andreev reflection (AR) in single- and multi-interface superconducting

\footnotetext{
*yctao88@163.com

†qifenghua.hi@163.com
}

Published by the American Physical Society under the terms of the Creative Commons Attribution 4.0 International license. Further distribution of this work must maintain attribution to the author(s) and the published article's title, journal citation, and DOI. hybrid junctions [27-32]. The interface between a superconductor (S) and a metal may reflect a negatively charged electron from the metal side as a positively charged hole, while the missing charge of $2 e$ enters the $\mathrm{S}$ as an electron pair. This process determines the conductance of the junction at bias voltages below the superconducting gap because it is the mechanism that converts a normal dissipative current into a dissipationless supercurrent. In addition, the interplay of superconductivity and ferromagnetism in hybrid junctions can lead to intriguing phenomena [32-35]. For instance, the novel AR could appear in a clean ferromagnet/ferromagnet/S (FFS) junction due to noncollinear magnetizations in the two F layers. Specifically, the incident electron and the Andreev reflected hole come from the same spin subband resulting in spin-triplet pairing states, which was introduced by Niu and Xing [33]. Afterward, Zhang studied the transport properties of graphene-based FFS junctions with noncollinear magnetizations [34]. He found that for an exchange energy larger than the superconducting gap, the novel AR manifests itself as subgap differential conductance peaks because of the formation of spin-flipped Andreev bound states in the intermediate F layer. Recently, Beirenvand et al. proposed a graphenebased superconducting hybrid junction comprised of F, RSO, and $\mathrm{S}$ in which RSO stands for a region with a Rashba spin-orbit interaction [35]. They revealed that equal- and opposite-spin pair correlations exist near the F-RSO interface and demonstrated that a direct link of the novel AR and equalspin pairings arose by the proximity effect in the presence of a RSO interaction.

However, due to linear energy dispersion at the Dirac point of graphene, the subgap conductance originates from a mix of opposite- and equal-spin pairing states in previous works, which makes it very difficult to modulate the opposite- or equal-spin pairing correlations individually. Moreover, the previous research on silicene-based superconducting hybrid 


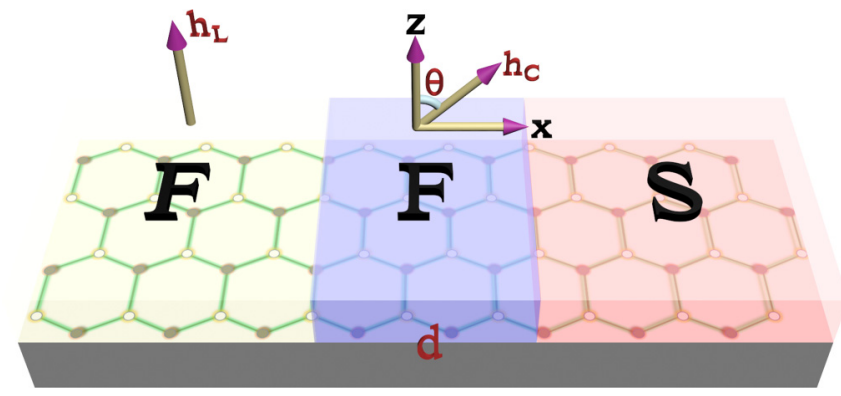

FIG. 1. Schematic of a silicene-based FFS junction with noncollinear magnetizations.

junctions is deficient in studying the spin-triplet pairing correlation, which can be attributed to the lack of noncollinear magnetizations in the corresponding junctions [24-26].

Therefore, it is highly desirable to fabricate and separate the opposite- and equal-spin pairings in transport processes. Motivated by this purpose and the spin-orbit coupling-induced energy gap of silicene, in this paper, we investigate the modulation between spin pairing states and the corresponding subgap conductance in a silicene-based FFS superconducting hybrid structure with noncollinear magnetizations in the two F layers. In our proposed setup, the separation of oppositeand equal-spin pairings is realized by tuning the gate voltage in perpendicular magnetization. Particularly, the electron and resultantly Andreev reflected hole can be fully spin polarized. The separation of fully spin-polarized oppositeand equal-spin pairing states results in the corresponding subgap conductance originating from either conventional or novel AR. This behavior shows distinctly different behavior from that of previous works [33-35], in which the conductance results from both conventional and novel AR. We also investigate the influence of the magnitude and orientation of the exchange field in the central region on the pairing states and then the corresponding subgap conductance. It is demonstrated that the fully spin-polarized equal-spin pairing correlation is enhanced with an increasing exchange field of the central region. Furthermore, the length of the central region can be large for the formation of fully spin-polarized equal-spin pairing states, but not for the opposite-spin pairing ones. Our work provides a feasible platform for exploring fully spin-polarized opposite- and equal-spin pairing states individually and their corresponding transport properties.

\section{MODEL}

We consider a silicene-based FFS junction with noncollinear magnetizations, as illustrated in Fig. 1, where a silicene sheet is deposited on the substrate in the $x-y$ plane, with $\mathrm{F}$ and $\mathrm{S}$ representing proximity-induced ferromagnetic and superconducting layers, respectively. Here, $\boldsymbol{h}_{L}$ and $\boldsymbol{h}_{C}$ respectively denote the exchange fields in the left and central F layers, and $d$ is the length of the central region. To explore the transport properties of the junction, we first begin with the tight-binding Hamiltonian of this system expressed as [36-39]

$$
H_{T}=H_{F}+H_{S} .
$$

Here, $H_{F}$ and $H_{S}$ are the Hamiltonians of the ferromagnetic and superconducting layers, respectively. They have the following forms,

$$
\begin{aligned}
& H_{F}=H_{0}+\sum_{m, \alpha, \beta, \kappa} c_{i \alpha}^{\dagger}\left(\boldsymbol{\sigma} \cdot \boldsymbol{h}_{\kappa}\right) c_{i \beta}+\sum_{m, \alpha, \kappa} \mu_{\kappa} c_{m, \alpha, \kappa}^{\dagger} c_{m, \alpha, \kappa}, \\
& H_{S}=H_{0}+\sum_{m} \Delta\left(c_{m \uparrow}^{\dagger} c_{m \downarrow}^{\dagger}+c_{m \downarrow} c_{m \uparrow}\right)+\sum_{m, \alpha} \mu_{S} c_{m \alpha}^{\dagger} c_{m \alpha},
\end{aligned}
$$

where the zero external-field Hamiltonian is $[3,5,24]$

$$
H_{0}=-t_{0} \sum_{\langle m, n\rangle, \alpha} c_{m \alpha}^{\dagger} c_{n \alpha}+\frac{i \lambda}{3 \sqrt{3}} \sum_{\langle\langle m, n\rangle\rangle, \alpha, \beta} v_{m n} c_{m \alpha}^{\dagger} \sigma_{\alpha \beta}^{z} c_{n \beta} .
$$

In Eqs. (2)-(4), $c_{m \alpha}\left(c_{m \alpha}^{\dagger}\right)$ is the annihilation (creation) operator at site $m$ with spin $\alpha . \boldsymbol{h}_{\kappa}$ represents the exchange field in region $\kappa(\kappa=L$ for the left region and $\kappa=C$ for the central region). In this paper, the exchange field will be chosen as $\boldsymbol{h}_{L}=\left(0,0, h_{L}\right)$ and $\boldsymbol{h}_{C}=\left(h_{C} \sin \theta, 0, h_{C} \cos \theta\right)$. $\mu_{\kappa}=\mu_{\kappa 0}+e V_{\kappa}$ with $\mu_{\kappa 0}$ the chemical potential is the electrochemical potential (Fermi level) of the two ferromagnetic layers, which can be tuned by gate voltage $e V_{\kappa}[24,25,40,41]$. $t_{0}=1.6 \mathrm{eV}$ denotes the nearest-neighbor hopping energy, $\lambda=3.9 \mathrm{meV}$ indicates the effective spin-orbit coupling parameter, and $\boldsymbol{\sigma}=\left(\sigma_{x}, \sigma_{y}, \sigma_{z}\right)$ is the Pauli matrix of spin. $\langle m, n\rangle$ and $\langle\langle m, n\rangle\rangle$ represent the summations over the nearestand next-nearest-neighboring lattice sites, respectively. $v_{m n}=$ 1 for anticlockwise next-nearest-neighboring hopping and $v_{m n}=-1$ for clockwise hopping with respect to the silicene sheet. In order to ensure the validity of the mean-field approximation, the Fermi energy $\mu_{S}$ in the $S$ region should be larger than the superconducting gap $\Delta[24,25]$. Therefore, the superconducting pairing potential $\Delta$ and Fermi energy of the superconducting layer $\mu_{S}$ are respectively set as $2 \mathrm{meV}$ and $0.03 \mathrm{eV}$ in the following.

With a combination of the two kinds of main contributed transport processes, the subgap conductance can be written as [36-39]

$$
\begin{aligned}
g= & g_{\mathrm{AR}}+g_{\mathrm{NAR}} \\
= & \frac{e}{h W} \sum_{k_{y}} \operatorname{Tr}\left[2 \Gamma_{L, e}^{\alpha} G_{e h, \alpha \bar{\alpha}}^{r} \Gamma_{L, h}^{\bar{\alpha}} G_{e h, \alpha \bar{\alpha}}^{a}\right. \\
& \left.+2 \Gamma_{L, e}^{\alpha} G_{e h, \alpha \alpha}^{r} \Gamma_{L, h}^{\alpha} G_{e h, \alpha \alpha}^{a}\right],
\end{aligned}
$$

where $g_{\mathrm{AR}}$ and $g_{\mathrm{NAR}}$ respectively represent the subgap conductance originating from the conventional and novel ARs. $k_{y}=2 \pi n / W$ is the transverse momentum with $n$ being an integer to indicate the channels and $W$ denoting the transverse width of the structures. $\Gamma_{L(R), e(h)}^{\alpha(\bar{\alpha})}$ is a block of the linewidth function $\Gamma_{L(R)}=i\left(\Sigma_{L(R)}^{r}-\Sigma_{L(R)}^{a}\right)$ with retarded/advanced self-energy $\Sigma_{L(R)}^{r(a)}$. Here, $e(h)$ labels the electron (hole) component of the Nambu space, $L(R)$ stands for the left (right) region, and the spin $\bar{\alpha}$ is opposite to spin $\alpha . g_{L(R)}^{r(a)}$ is the retarded (advanced) surface Green's function, which can be calculated numerically by the recursive method $[42,43] . G_{e h}^{r(a)}$ is the matrix block of retarded (advanced) Green's function of the central region which is determined by $G^{r(a)}=$ $\left(E I-H_{S}-\Sigma_{L}^{r(a)}-\Sigma_{R}^{r(a)}\right)^{-1}$. 
(a)

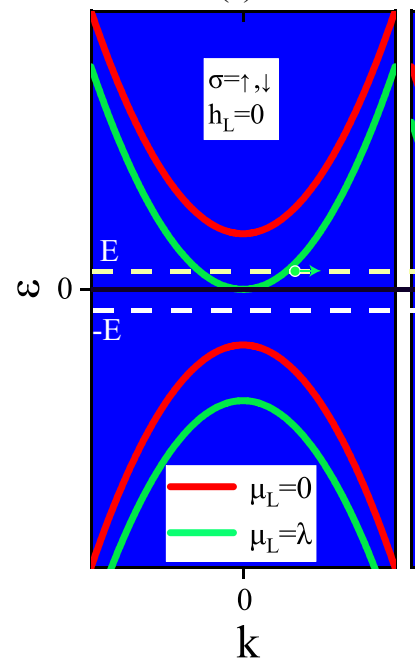

(b)

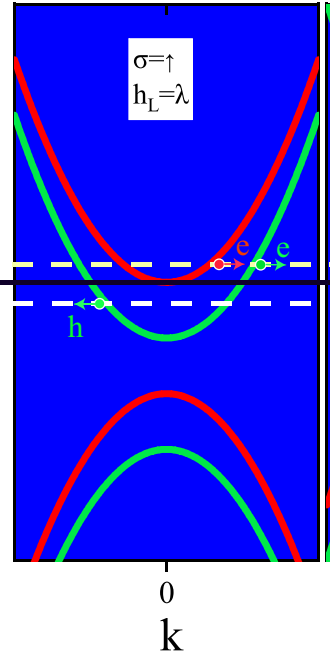

(c)

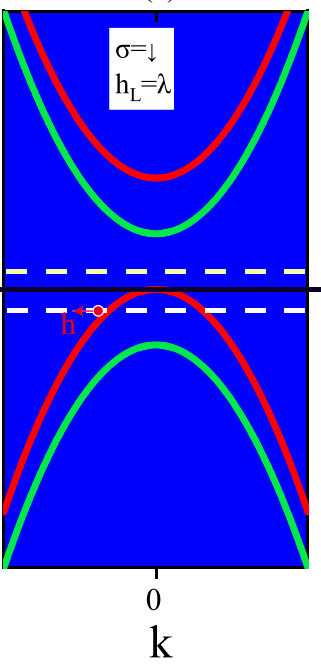

(d)

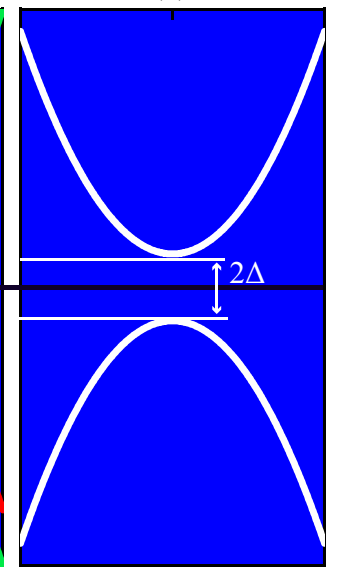

FIG. 2. Band structures of (a)-(c) the left $\mathrm{F}$ region (sharing the same bands with the central $\mathrm{F}$ region) and (d) $\mathrm{S}$ region, where $\mu_{L}$ is the Fermi level, $h_{L}$ denotes the magnitude of exchange field, $E$ is the applied bias voltage, $2 \Delta$ stands for the superconducting gap, $\sigma=\uparrow,(\downarrow)$ indicates spin up (spin down), and $e(h)$ labels the electron (hole).

\section{RESULTS AND DISCUSSIONS}

\section{A. Band structures and switch effect between fully} spin-polarized opposite- and equal-spin pairings

The electronic band structure can provide perspicuous insights into the properties of multiple backscatterings and transport processes. To obtain the band structure of the left F region conveniently, we need to transform Eq. (2) into the momentum space form as given below [24,25],

$$
H_{\eta, \sigma}^{e(h)}=\hbar v_{F}\left(\eta k_{x} \tau_{x}-k_{y} \tau_{y}\right)+\eta \sigma \lambda \tau_{z}-(+) \mu_{L}-\sigma h_{L},
$$

which is valid for low-energy excitation. The corresponding parameters are defined as follows: $v_{F}=3 a t_{0} / 2 \hbar$ is the Fermi velocity with the lattice constant $a=3.86 \AA, \eta=1(-1)$ labels the $K\left(K^{\prime}\right)$ valley, $k_{x}$ and $k_{y}$ are the wave vectors in the $x$ and $y$ directions, $\boldsymbol{\tau}=\left(\tau_{x}, \tau_{y}, \tau_{z}\right)$ is the Pauli matrix denoting the sublattice pseudospin, $\tau_{0}$ is a $2 \times 2$ unit matrix, and $\sigma= \pm 1$ indicates the spin index. By solving the eigenvalue of Eq. (6), we can acquire the dispersion relation as follows [25],

$$
\varepsilon_{\sigma}^{e(h)}= \pm \sqrt{\left(\hbar v_{F} k\right)^{2}+\lambda^{2}}-(+) \mu_{L}-\sigma h_{L} .
$$

Figure 2 illustrates the low-energy excitation spectrum. As we can see, there is an intrinsic energy gap $2 \lambda$ of silicene resulting from the effective spin-orbit coupling. For vanishing field $h_{L}=0$ and Fermi level $\mu_{L}=0$, the incident energy $E$ resides in the energy gap as depicted in Fig. 2(a), which means there is no electron incident from the left $F$ layer and then the subgap conductance vanishes. By tuning the Fermi level $\mu_{L}$ to $\lambda$, the incident energy $E$ crosses the conduction band. Consequently, in the presence of noncollinear magnetizations, for an incident electron with spin $\sigma$ from the conduction band, the reflected electron would be spin $\sigma$ or $\bar{\sigma}$ (opposite to $\sigma$ ), and there are no Andreev reflected holes due to the energy of the hole residing in the energy gap, where the subgap conductance is also nonexistent.

Nevertheless, with the existing exchange field, the energy band will split into spin-up and spin-down subbands. For the exchange field $h_{L}=\lambda$ and $\mu_{L}=0$, at the applied bias voltage $E \in[0, \Delta]$, the incident electron only comes from the spin-up subband, and the Andreev reflected hole must be spin-down as a result of the vanishing states of the spin-up hole, as shown in Figs. 2(b) and 2(c). In this electron-hole conversion, the electron and hole come from opposite spin subbands, i.e., opposite-spin pairing states, which is corresponds to conventional AR. Here, the electron and hole are fully spin-up and spin-down polarized, respectively, meaning fully spin-polarized opposite-spin pairing states. The corresponding normalized subgap conductance $g / g_{0}$ vs $E$ is plotted in Fig. 3(a), in which $g_{0}$ is the conductance of a uniform silicene sheet at the same bias voltage $E$, the magnitude and orientation of the exchange field in the central region are respectively fixed at $h_{C}=\lambda$ and $\theta=\pi / 2$, the Fermi level of the central region is chosen as $\mu_{C}=0$, and the length of the central region is fixed at $d=0.2 \xi$ with $\xi=\hbar v_{F} / \Delta$ denoting the superconducting coherent length. Under this situation, the subgap conductance is attributed to the conventional AR. Interestingly, we can find a zero-bias conductance dip with the value being zero, owing to the completely suppressed AR. This behavior could originate from the noncollinear magnetizations and vanishing density of states of the spin-up electron and spin-down hole at zero-bias voltage.

Subsequently, by tuning the Fermi level $\mu_{L}=\lambda$, it occurs that the energies of the electron $E$ and hole $-E$ both only cross the spin-up subband, as shown in Fig. 2 (green mark). In this case, for the magnetizations perpendicular to each other, the novel AR emerges that the incident spin-up electron could be reflected as a spin-up hole, indicating equal-spin pairing or spin-triplet pairing states, as discussed in Refs. [33,35]. It is worth noting that the electron and hole are both spin-up polarized, which indicates that the perfect fully spin-polarized equal-spin pairing state does not have any contamination from opposite-spin pairing states. The corresponding subgap conductance spectrum is plotted in Fig. 3(b), where $h_{C}=\lambda, \mu_{C}=\lambda$, and $\theta=\pi / 2$. As a result of the existence of spin-triplet states, we can find the nonvanished zero-bias conductance dip. 

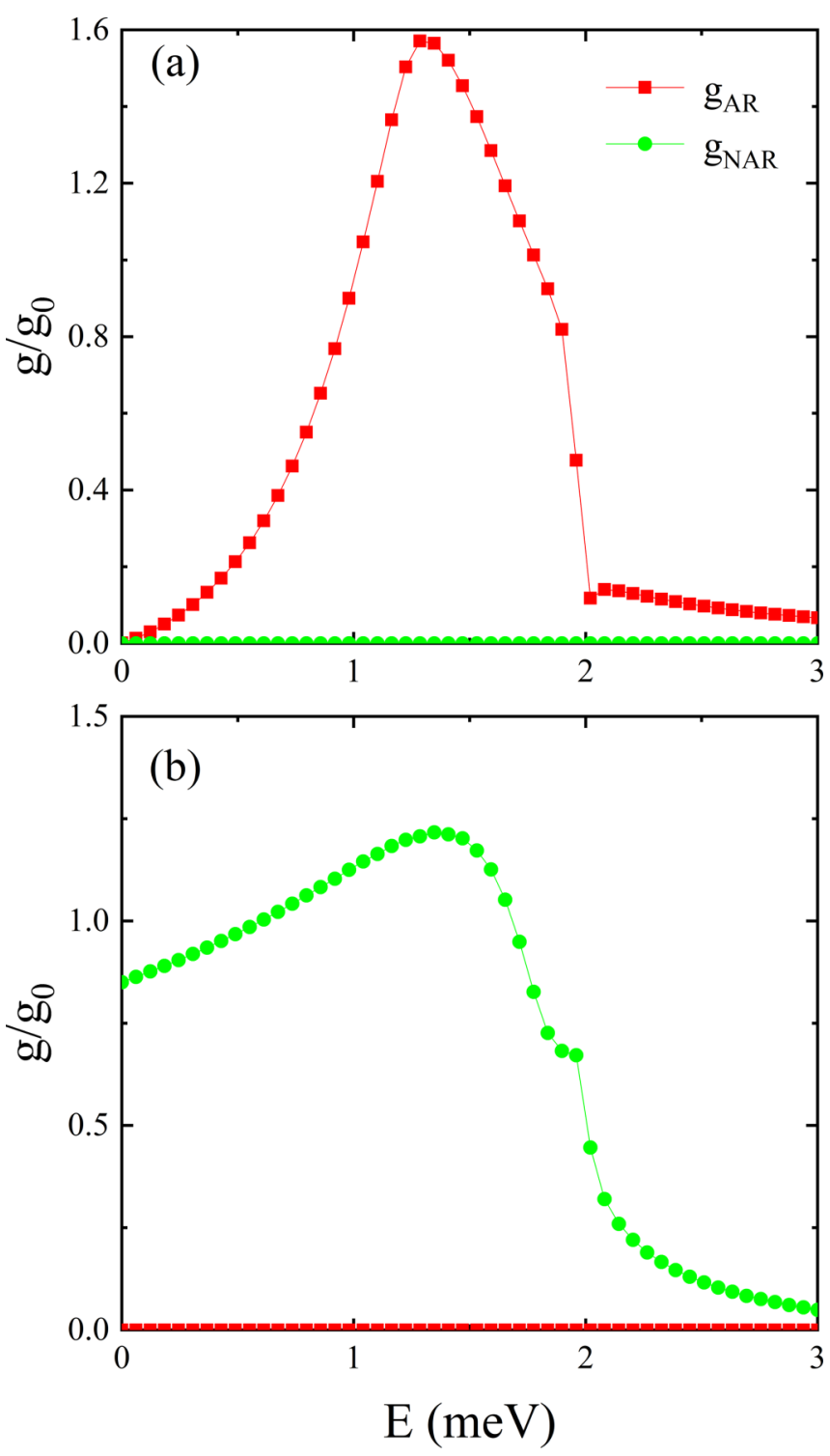

FIG. 3. The subgap conductance $g / g_{0}$ vs the applied voltage $E$, where $g_{\mathrm{AR}}$ and $g_{\mathrm{NAR}}$ represent the conventional and novel AR-induced conductance, respectively. For (a), $\mu_{L}=\mu_{C}=0$ and $h_{L}=\lambda$, for (b), $\mu_{L}=\mu_{C}=\lambda$ and $h_{L}=\lambda$, and the other relevant parameters are chosen as $\theta=\pi / 2, h_{C}=\lambda, d=0.2 \xi$, the superconducting pairing potential $\Delta=2 \mathrm{meV}$, and the Fermi energy of $\mathrm{S}$ region $\mu_{S}=0.03 \mathrm{eV}$.

These behaviors are distinctly different from those of the previous works [32-34], where the opposite- and equal-spin pairing states are mixed. However, in our device, the subgap conductance either originates from conventional or novel AR. Our findings also suggest the electron-hole spin pairing valve, in which the fully spin-polarized opposite- and equal-spin pairing states can be interswitched by tuning the gate voltage.

\section{B. Modulation of fully spin-polarized opposite- or equal-spin pairing}

To further understand the influence of fully spin-polarized opposite- and equal-spin pairing correlations on the subgap conductance, it is of fundamental significance to explore the

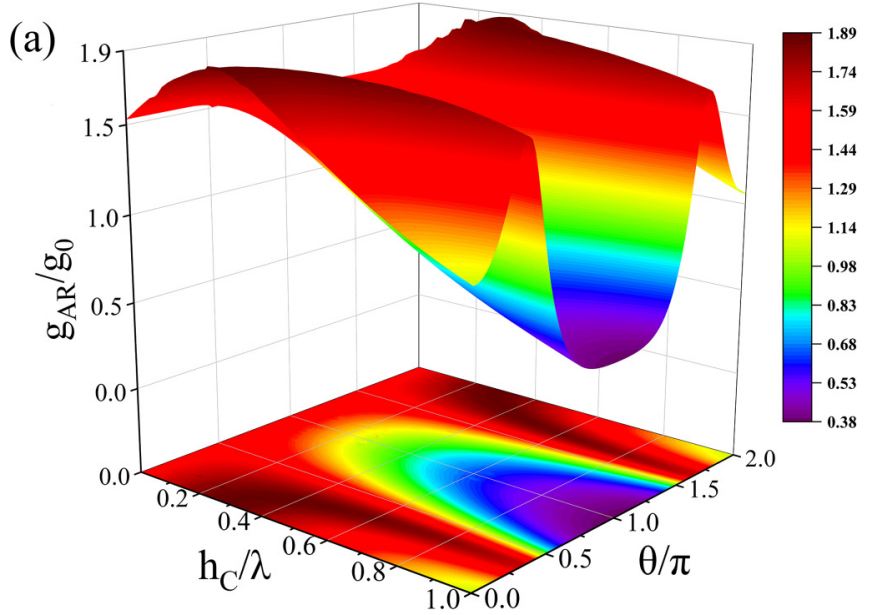

(b)

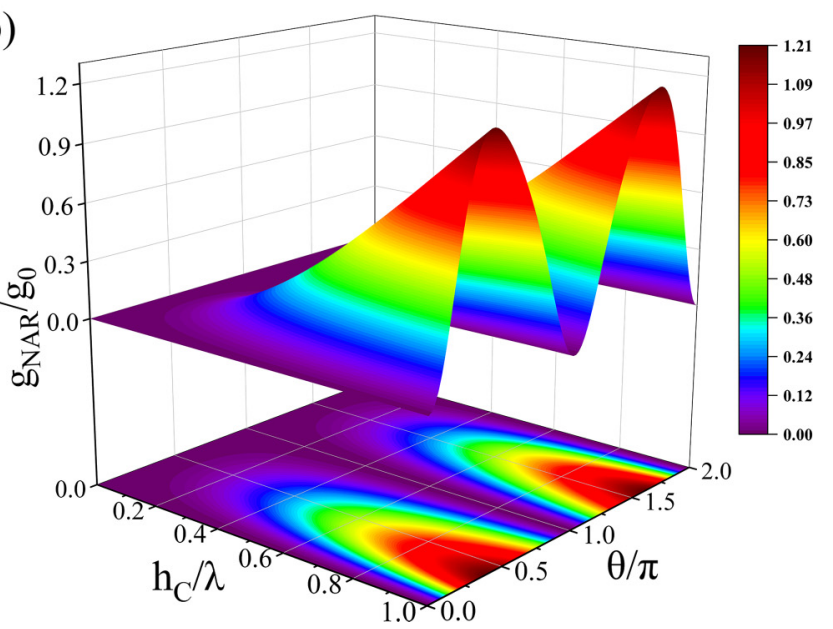

FIG. 4. (a) and (b) are respectively the conventional and novel AR-induced subgap conductance as functions of the magnitude $h_{C}$ and orientation $\theta$ of the exchange field in the central region at $E=$ $1 \mathrm{meV}$. The other related parameters for (a) and (b) are the same as those in Figs. 3(a) and 3(b).

process of electron-hole conversion and the formation of equal-spin pairing states. Therefore, we study the effect of magnitude $h_{C}$ and orientation $\theta$ of the exchange field in the central region on conventional and novel AR-induced subgap conductance. The subgap conductance as a function of $h_{C}$ and $\theta$ is plotted in Fig. 4. Figure 4(a) exhibits a subgap conductance resulting from the conventional AR, where the Fermi level is fixed at $\mu_{L}=\mu_{C}=0$ and the exchange field in the left region $h_{L}=\lambda$. For $\theta=\pi$, meaning the opposite magnetization orientations in the two $F$ layers, the conductance dip always appears and the value of the dip monotonously decreases with increasing $h_{C}$. We can also find the conductance peak (dark red region), where the conventional AR resonant condition is satisfied due to the resonant opposite-spin pairing correlation.

The novel AR-induced subgap conductance (corresponding to fully spin-polarized equal-spin pairing) modulated by $\boldsymbol{h}_{C}$ is exhibited in Fig. 4(b), where $\mu_{L}=\mu_{C}=\lambda$ and $h_{L}=\lambda$. For fixed $\theta$, the subgap conductance is increased 

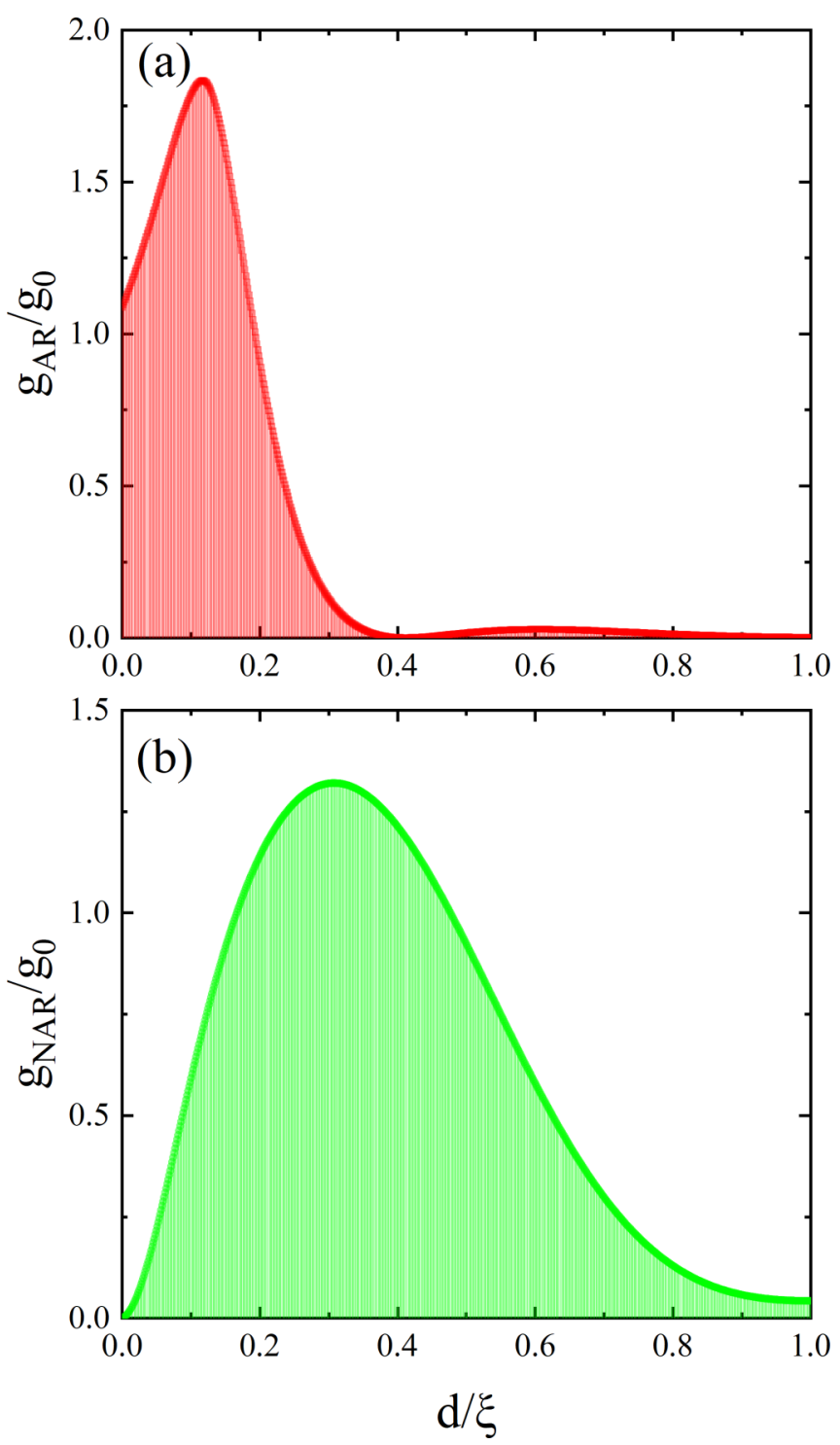

FIG. 5. The subgap conductances (a) $g_{\mathrm{AR}} / g_{0}$ and (b) $g_{\mathrm{NAR}} / g_{0}$ vs the length of central region $d$ for $E=1 \mathrm{meV}$. The other corresponding parameters for (a) and (b) are the same as those in Figs. 3(a) and 3(b).

with an enhancement of the exchange field $h_{C}$, which is different from the behavior of conventional AR-induced subgap conductance. In addition, at around $\theta=\pi / 2$ and $3 \pi / 2$, the conductance peaks appear, which could be ascribed to the resonant equal-spin pairing state. In stark contrast with opposite-spin pairing, the fully spin-polarized equal-spin pairing state disappears at $h_{C}=0$, leading to the vanished novel AR and then subgap conductance. The subgap conductance also vanishes at $\theta=0$ or $\pi$ corresponding to parallel or antiparallel magnetic configurations, which can be also attributed to the vanished spin-triplet pairing states.

To get profound insight into the properties of fully spinpolarized electron-hole conversion, we investigate how the size of the central region influences the subgap conductance. Figure 5(a) shows the conventional AR-induced subgap con- ductance as a function of the length of the central region at applied bias voltage $E=1 \mathrm{meV}$, where $h_{L}=h_{C}=\lambda, \mu_{L}=$ $\mu_{C}=0$, and $\theta=\pi / 2$. For $d=0$, the configuration corresponds to the FS structure and the subgap conductance is nonzero. With $d$ increased to $0.4 \xi$, the conductance first increases to maximum and then sharply drop to zero. For $0.4<d / \xi<1$, the conductance is always around zero, which indicates that the fully spin-polarized opposite-spin correlation would be suppressed due to the noncollinear magnetizations at the long central region.

The conductance resulting from novel AR versus the length of the central region at $E=1 \mathrm{meV}$ is plotted in Fig. 5(b), where the parameters are chosen as $h_{L}=h_{C}=\lambda, \mu_{L}=\mu_{C}=$ $\lambda$, and $\theta=\pi / 2$. In contrast with conventional AR-induced subgap conductance, novel AR-induced subgap conductance is zero at $d=0$. It occurs because of the disappearance of noncollinear magnetizations and then the fully spin-polarized equal-spin pairing states. Nevertheless, the novel AR-induced conductance reaches a maximum at $d=0.3 \xi$ and gradually decreases with enlarging the size of the central region. It is worth noticing that the conductance does not reduce to zero at a long central layer. These behaviors manifest that the large length of the central region can be taken for the formation of fully spin-polarized equal-spin pairing states but not for fully spin-polarized opposite-spin pairing.

\section{CONCLUSION}

In conclusion, we have studied the modulation between opposite- and equal-spin pairings and then conventional and novel AR-induced transport properties of silicene-based FFS superconducting hybrid junctions with noncollinear magnetizations. Due to the presence of noncollinear magnetizations and the gapped energy band of silicene, three peculiar features are exhibited. First, pure fully spin-polarized equal-spin pairing states without any contamination from opposite-spin pairing states can be achieved, in stark contrast to previous works, where the opposite- and equal-spin pairings are mixed. Second, the switch effect between fully spin-polarized opposite- and equal-spin pairings is realized by tuning the gate voltage. Third, novel AR-induced subgap conductance is increased with the enhancement of the exchange field of the central region in noncollinear magnetizations, which originates from the enhanced fully spin-polarized equal-spin pairing correlation. Lastly, for the formation of fully spinpolarized equal-spin pairing states, the length of the central region in perpendicular magnetizations can be taken to be large, but not for fully spin-polarized opposite-spin pairing. Therefore, our findings offer a practicable platform for investigating the properties of opposite- and equal-spin pairings separately.

\section{ACKNOWLEDGMENTS}

This work was supported by the National Natural Science Foundation of China under Grants No. 11874221 and No. 11805103, and Jiangsu Natural Science Foundation (Grant No. BK20190137). 
[1] A. H. Castro Neto, F. Guinea, N. M. R. Peres, K. S. Novoselov, and A. K. Geim, The electronic properties of graphene, Rev. Mod. Phys. 81, 109 (2009).

[2] M. Z. Hasan and C. L. Kane, Colloquium: Topological insulators, Rev. Mod. Phys. 82, 3045 (2010).

[3] J. E. Yang, X. L. Lü, C. X. Zhang, and H. Xie, Topological spinvalley filtering effects based on hybrid silicene-like nanoribbons, New J. Phys. 22, 053034 (2020).

[4] M. Ezawa, Monolayer topological insulators: Silicene, germanene, and stanene, J. Phys. Soc. Jpn. 84, 121003 (2015).

[5] C. C. Liu, H. Jiang, and Y. Yao, Low-energy effective Hamiltonian involving spin-orbit coupling in silicene and twodimensional germanium and tin, Phys. Rev. B 84, 195430 (2011).

[6] B. Lalmi, H. Oughaddou, H. Enriquez, A. Kara, S. Vizzini, B. Ealet, and B. Aufray, Epitaxial growth of a silicene sheet, Appl. Phys. Lett. 97, 223109 (2010).

[7] P. D. Padova, C. Quaresima, C. Ottaviani, P. M. Sheverdyaeva, and P. Moras, Evidence of graphene-like electronic signature in silicene nanoribbons, Appl. Phys. Lett. 96, 261905 (2010).

[8] P. Vogt, P. De Padova, C. Quaresima, J. Avila, E. Frantzeskakis, M. C. Asensio, A. Resta, B. Ealet, and G. Le Lay, Silicene: Compelling Experimental Evidence for Graphenelike Two-Dimensional Silicon, Phys. Rev. Lett. 108, 155501 (2012).

[9] S. Cahangirov, M. Topsakal, E. Aktürk, H. Sahin, and S. Ciraci, Two- and One-Dimensional Honeycomb Structures of Silicon and Germanium, Phys. Rev. Lett. 102, 236804 (2009).

[10] M. Ezawa, A topological insulator and helical zero mode in silicene under an inhomogeneous electric field, New J. Phys. 14, 033003 (2012).

[11] G. G. Guzmán-Verri and L. C. L. Y. Voon, Electronic structure of silicon-based nanostructures, Phys. Rev. B 76, 075131 (2007).

[12] C.-C. Liu, W. Feng, and Y. Yao, Quantum Spin Hall Effect in Silicene and Two-Dimensional Germanium, Phys. Rev. Lett. 107, 076802 (2011).

[13] N. D. Drummond, V. Zólyomi, and V. I. Falko, Electrically tunable band gap in silicene, Phys. Rev. B 85, 075423 (2012).

[14] N. Johnson, P. Vogt, A. Resta, P. D. Padova, I. Perez, D. Muir, E. Z. Kurmaev, G. L. Lay, and A. Moewes, The metallic nature of epitaxial silicene monolayers on Ag (111), Adv. Funct. Mater. 24, 5253 (2014).

[15] A. Fleurence, R. Friedlein, T. Ozaki, H. Kawai, Y. Wang, and Y. Yamada-Takamura, Experimental Evidence for Epitaxial Silicene on Diboride Thin Films, Phys. Rev. Lett. 108, 245501 (2012).

[16] L. Meng, Y. Wang, L. Zhang, S. Du, R. Wu, L. Li, Y. Zhang, G. Li, H. Zhou, W. A. Hofer, and H.-J. Gao, Buckled silicene formation on Ir (111), Nano Lett. 13, 685 (2013).

[17] D. Chiappe, E. Scalise, E. Cinquanta, C. Grazianetti, B. Broek, M. Fanciulli, M. Houssa, and A. Molle, Two-dimensional Si nanosheets with local hexagonal structure on a $\mathrm{MoS}_{2}$ surface, Adv. Mater. 26, 2096 (2014).

[18] I. Zutić, J. Fabian, and S. Das Sarma, Spintronics: Fundamentals and applications, Rev. Mod. Phys. 76, 323 (2004).

[19] W.-F. Tsai, C.-Y. Huang, T.-R. Chang, H. Lin, H.-T. Jeng, and A. Bansil, Gated silicene as a tunable source of nearly $100 \%$ spin-polarized electrons, Nat. Commun. 4, 1500 (2013).
[20] Y. Wang, J. Zheng, Z. Ni, R. Fei, Q. Liu, R. Quhe, C. Xu, J. Zhou, Z. Gao, and J. Lu, Half-metallic silicene and germanene nanoribbons: Towards high-performance spintronics device, Nano 7, 1250037 (2012).

[21] Y. Wang, R. Quhe, D. Yu, and J. Lu, Silicene spintronics - A concise review, Chin. Phys. B 24, 087201 (2015).

[22] S. Rachel and M. Ezawa, Giant magnetoresistance and perfect spin filter in silicene, germanene, and stanene, Phys. Rev. B 89, 195303 (2014).

[23] L. Tao, E. Cinquanta, D. Chiappe, C. Grazianetti, M. Fanciulli, M. Dubey, A. Molle, and D. Akinwande, Silicene field-effect transistors operating at room temperature, Nat. Nanotechnol. 10, 227 (2015).

[24] J. Linder and T. Yokoyama, Superconducting proximity effect in silicene: Spin-valley-polarized Andreev reflection, nonlocal transport, and supercurrent, Phys. Rev. B 89, 020504(R) (2014).

[25] H. Li, Subgap transport in silicene-based superconducting hybrid structures, Phys. Rev. B 94, 075428 (2016).

[26] Y. J. Wei and Y. C. Tao, Tunable spin-valley polarized transport channel in silicene-based superconducting hybrid structures, J. Appl. Phys. 129, 043902 (2021).

[27] A. F. Andreev, The thermal conductivity of the intermediate state in superconductors, Zh. Eksp. Teor. Fiz. 46, 1823 (1964) [Sov. Phys. JETP 19, 1228 (1964)].

[28] B. Panneitier and H. Courtois, Andreev reflection and proximity effect, J. Low Temp. Phys. 118, 599 (2000).

[29] G. Deutscher, Andreev-Saint-James reflections: A probe of cuprate superconductors, Rev. Mod. Phys. 77, 109 (2005).

[30] C. W. J. Beenakker, Colloquium: Andreev reflection and Klein tunneling in graphene, Rev. Mod. Phys. 80, 1337 (2008).

[31] C. W. J. Beenakker, Specular Andreev Reflection in Graphene, Phys. Rev. Lett. 97, 067007 (2006).

[32] F. S. Bergeret, A. F. Volkov, and K. B. Efetov, Odd triplet superconductivity and related phenomena in superconductorferromagnet structures, Rev. Mod. Phys. 77, 1321 (2005).

[33] Z. P. Niu and D. Y. Xing, Spin-Triplet Pairing States in Ferromagnet/Ferromagnet/d-Wave Superconductor Heterojunctions with Noncollinear Magnetizations, Phys. Rev. Lett. 98, 057005 (2007).

[34] Z. Y. Zhang, Novel Andreev reflection and differential conductance of a ferromagnet/ferromagnet/superconductor junction on graphene, J. Phys.: Condens. Matter 21, 095302 (2009).

[35] R. Beiranvand, H. Hamzehpour, and M. Alidoust, Tunable anomalous Andreev reflection and triplet pairings in spin-orbitcoupled graphene, Phys. Rev. B 94, 125415 (2016).

[36] J. Wang, L. Zhang, and K. S. Chan, Tunneling conductance of a magnetized zigzag graphene nanoribbon/superconductor junction, Phys. Rev. B 83, 125425 (2011).

[37] J. Wang and S. Liu, Crossed Andreev reflection in a zigzag graphene nanoribbon-superconductor junction, Phys. Rev. B 85, 035402 (2012).

[38] F. H. Qi, J. Cao, and G. J. Jin, Valley-controlled nonlocal transistor based on irradiated and biased bilayer graphene, Phys. Rev. B 98, 045422 (2018).

[39] P. Burset, A. Levy Yeyati, and A. Martín-Rodero, Microscopic theory of the proximity effect in superconductor-graphene nanostructures, Phys. Rev. B 77, 205425 (2008).

[40] K. S. Novoselov, A. K. Geim, S. V. Morozov, D. Jiang, Y. Zhang, S. V. Dubonos, and I. V. Grigorieva, Electric 
field effect in atomically thin carbon films, Science 306, 666 (2004).

[41] Z. Q. Zhang, C. Z. Chen, Y. Wu, H. Jiang, J. Liu, Q. F. Sun, and X. C. Xie, Chiral interface states and related quantized transport in disordered Chern insulators, Phys. Rev. B 103, 075434 (2021).
[42] D. H. Lee and J. D. Joannopoulos, Simple scheme for surfaceband calculations. II. The Green's function, Phys. Rev. B 23 , 4997 (1981).

[43] M. P. Lopez et al., Quick iterative scheme for the calculation of transfer matrices: Application to Mo (100), J. Phys. F: Met. Phys. 14, 1205 (1984). 